\title{
Beyond Born-Oppenheimer approximation in ultracold atomic collisions
}

\author{
Eberhard Tiemann $\odot,{ }^{*}$ Philipp Gersema $\odot$, Kai K. Voges $\odot$, Torsten Hartmann $\odot$, Alessandro Zenesini $\odot$, and Silke Ospelkaus ${ }^{\dagger}$ \\ Institut für Quantenoptik, Leibniz Universität Hannover, 30167 Hannover, Germany
}

(Received 17 December 2019; accepted 4 March 2020; published 26 March 2020)

\begin{abstract}
We report on deviations beyond the Born-Oppenheimer approximation in potassium interatomic potentials. Identifying three previously unknown $d$-wave Feshbach resonances, we significantly improve the understanding of the ${ }^{39} \mathrm{~K}$ interatomic potentials. Combining these observations with the most recent data on known inter- and intraisotope Feshbach resonances, we show that Born-Oppenheimer corrections can be determined from atomic collisional properties alone and that significant differences between the homo- and heteronuclear cases appear.
\end{abstract}

DOI: 10.1103/PhysRevResearch.2.013366

\section{INTRODUCTION}

In quantum chemistry and molecular physics, the assumption that the electronic and nuclear motions can be separately treated is well justified by the three orders of magnitude separating the proton and the electron masses. The nuclei are considered as fixed objects at relative distance $R$ when solving the eigenvalue problem of the electron motion, resulting in a $R$-dependent electronic energy, which is taken as the potential for the nuclear motion. This approximation leads to large simplifications when solving the Schrödinger equation for molecules and is named Born-Oppenheimer approximation (BOA) [1]. The BOA is extremely powerful in matching theoretical predictions and spectroscopic results, particularly concerning the understanding of diatomic molecules. One major aspect within the BOA is that the same interatomic potential (BO potential) is used for different isotopes by simply rescaling the nuclear motion according to the reduced molecular mass. Deviations from this assumption lead to perturbative corrections to the BOA on the order of the electron-to-proton mass ratio. The isotopic dependence of corrections has been discussed in many papers; see, for example, Refs. [2-4]. These deviations from the BO-potential approach correspond to shifts in energy levels on the order of $\Delta E / E \approx 10^{-4}$ or less and they have been observed in spectroscopy experiments like Refs. [5-7] and in the dissociation energy of different isotope combinations of hydrogen diatomic molecules [8].

Effects of the corrections to the BOA are much weaker at the collisional threshold of atom pairs as the long-range behavior of the interatomic potential is weakly affected by shortrange variations. Recent developments in molecule cooling and molecule association from ultracold atoms have considerably increased the experimental resolution, giving access

\footnotetext{
*tiemann@iqo.uni-hannover.de

†silke.ospelkaus@iqo.uni-hannover.de

Published by the American Physical Society under the terms of the Creative Commons Attribution 4.0 International license. Further distribution of this work must maintain attribution to the author(s) and the published article's title, journal citation, and DOI.
}

to study effects beyond the BOA. Corrections to the triplet and singlet scattering lengths are indeed predicted to be on the order of a few tenths of the Bohr radius $a_{0}$ [7] and are practically undetectable. However, Feshbach resonances are an effective passkey as they carry important information of the short-range potential to the atomic threshold [9]. A particular interesting case is given by the collisional properties of ultracold potassium atoms. Potassium features two stable bosonic isotopes $\left({ }^{39} \mathrm{~K}\right.$ and $\left.{ }^{41} \mathrm{~K}\right)$ and a very long living fermionic one $\left({ }^{40} \mathrm{~K}\right)$. All these isotopes have been cooled to quantum degeneracy both in single- and two-isotope experiments and collisional data for five $\left({ }^{39} \mathrm{~K}-{ }^{39} \mathrm{~K},{ }^{40} \mathrm{~K}-{ }^{40} \mathrm{~K},{ }^{41} \mathrm{~K}-{ }^{41} \mathrm{~K},{ }^{39} \mathrm{~K}-{ }^{41} \mathrm{~K}\right.$, ${ }^{40} \mathrm{~K}-{ }^{41} \mathrm{~K}$ ) of the six possible combinations are available in literature [10-14]. The comparison of Feshbach resonance positions for different isotope combinations is a promising way to reveal corrections to the BOA. First hints of such corrections were obtained by Falke et al. [7] studying the two cases ${ }^{39} \mathrm{~K}^{-39} \mathrm{~K}$ and ${ }^{40} \mathrm{~K}-{ }^{40} \mathrm{~K}$ available at that time. In this paper, we present the experimental observation of previously unmeasured $d$-wave Feshbach resonances of ${ }^{39} \mathrm{~K}$ and show how this allows us to improve the knowledge of ${ }^{39} \mathrm{~K}_{2}$. We combine this result with the published literature on potassium Feshbach resonances and we determine corrections to the BOA from collisional data alone.

The paper is structured as follows. In Sec. II, we explain how to reveal effects from beyond BOA corrections in atomic collisional properties. In Sec. III, we present the observation of three new Feshbach resonances for ${ }^{39} \mathrm{~K}$, which enhances the knowledge on ${ }^{39} \mathrm{~K}_{2}$ to use this dimer as reference for the full isotope analysis. In Sec. IV, we quantify the corrections to the BOA thanks to a multiparameter fit of the new and already known Feshbach resonance positions.

\section{THEORY ASPECTS}

To treat the collision of an atom pair of alkali atoms at low kinetic energy, we set up the Hamiltonian of the coupled system of the two lowest molecular states $X^{1} \Sigma_{g}^{+}$and $a^{3} \Sigma_{u}^{+}$, because the product state of two ground-state atoms is generally a mixture of singlet and triplet states. The appropriate Hamiltonian is presented in many papers, e.g., Refs. [7,9], and will not be repeated here. It contains the hyperfine 
interaction, also responsible for the singlet-triplet mixing, the atomic Zeeman interaction, and the effective spin-spin interaction of the two atoms in their doublet states. The nuclear motion is governed by the molecular potentials of the two interacting molecular states.

The potential functions within the Born-Oppenheimer approximation (BO potentials) are represented in analytic form as described in detail in Ref. [7] in three $R$ sections divided by an inner $R_{\text {in }}$ and outer radius $R_{\text {out }}$.

In the intermediate range around the minimum, it is described by a finite power expansion

$$
U_{\mathrm{IR}}(R)=\sum_{i=0}^{n} a_{i} \xi(R)^{i}
$$

with a nonlinear variable function $\xi$ of internuclear separation $R$ :

$$
\xi(R)=\frac{R-R_{m}}{R+b R_{m}} .
$$

In Eq. (1), the $\left\{a_{i}\right\}$ are fitting parameters and $b$ and $R_{m}$ are chosen such that only few parameters $a_{i}$ are needed for describing the steep slope at the short internuclear separation side and the smaller slope at the large $R$ side by the analytic form of Eq. (1). $R_{m}$ is normally close to the value of the equilibrium separation.

The potential is extrapolated for $R<R_{\text {in }}$ with

$$
U_{\mathrm{SR}}(R)=A+B / R^{N_{s}}
$$

by adjusting the $A$ and $B$ parameters to get a continuous transition at $R_{\text {in }}$; the final fit uses $N_{s}$ equal to 12 and 6 for $X^{1} \Sigma_{g}^{+}$and $a^{3} \Sigma_{u}^{+}$states, respectively, as adequate exponents.

For large internuclear distances $\left(R>R_{\text {out }}\right)$, we adopt the standard long-range form of molecular potentials:

$$
U_{\mathrm{LR}}(R)=U_{\infty}-\frac{C_{6}}{R^{6}}-\frac{C_{8}}{R^{8}}-\frac{C_{10}}{R^{10}} \pm E_{\mathrm{exch}}(R),
$$

where the exchange contribution is given by

$$
E_{\text {exch }}(R)=A_{\text {ex }} R^{\gamma} \exp (-\beta R)
$$

and $U_{\infty}$ is set to zero, which fixes the energy reference of the total potential scheme.

The BO potentials are extended by correction functions $U_{\text {ad }}(R)$, which make the full potentials mass dependent. These correction functions $[3,4,15]$ contain matrix elements of the nuclear momentum operators over the electronic wave functions of the considered electronic state and other ones with $\Delta \Omega=0$, where $\Omega$ is the projection of the total electronic angular momentum onto the molecular axis. $U_{\mathrm{ad}}(R)$ is the socalled adiabatic correction to the BO-potential function, and it contains the interaction of the considered electronic state with all states according the selection rule $\Delta \Omega=0$ by the nuclear vibrational motion. We do not include nonadiabatic correction with the selection rule $\Delta \Omega= \pm 1$ because it will be negligibly small for collisions with low partial waves as $s, p$, or $d$.

Watson [3] shows that in the lowest order the mass dependence of these corrections for a molecule $A B$ will be of the form $U_{A}(R) \frac{m_{e}}{M_{A}}+U_{B}(R) \frac{m_{e}}{M_{B}}$, where $M_{A(B)}$ is the atomic mass of atom $A(B)$ and $m_{e}$ is the electron mass. For true heteronuclear molecules, the coefficients $U_{A}(R)$ and $U_{B}(R)$ will be different; for homonuclear cases in the electronic system such as $\mathrm{K}_{2}$ both coefficients will be equal and thus the isotope dependence of the correction function will be inversely proportional to the reduced mass $\mu$ of the molecule. Van Vleck [15] considered the mass dependence of the heteronuclear cases in the hydrogen-deuterium (HD) molecule and found that the corrections should be extended by a term $\left(M_{A}-M_{B}\right)^{2} /\left(M_{A}+M_{B}\right)^{2}$. Thus, in our case with the nuclei like ${ }^{39} \mathrm{~K}-{ }^{41} \mathrm{~K}$, the representation of the correction functions should read

$$
U_{\mathrm{ad}}(R)=U_{\mathrm{gen}}(R) \frac{m_{e}}{\mu}+U_{\mathrm{asym}}(R) m_{e}\left(\frac{M_{A}-M_{B}}{M_{A}+M_{B}}\right)^{2},
$$

where $\mu$ is the reduced mass for the molecular rovibrational motion. $U_{\text {gen }}(R)$ and $U_{\text {asym }}(R)$ are functions of the internuclear separation $R$. The subscripts refer to the general and asymmetric contributions.

\section{III. $d$-WAVE FESHBACH RESONANCES IN ${ }^{39} \mathrm{~K}$}

In our setup, ultracold samples of ${ }^{39} \mathrm{~K}$ atoms are prepared by sympathetic cooling in a bath of evaporatively cooled $\mathrm{Na}$ atoms as explained in Refs. [16,17]. Compared to the experimental sequence used in our previous works, the mixture here is heavily unbalanced toward ${ }^{39} \mathrm{~K}$ and the $\mathrm{Na}$ atom number is practically negligible. During evaporation in a crossed optical dipole trap, the ${ }^{39} \mathrm{~K}$ atoms are initially in the $\left|f=1, m_{f}=-1\right\rangle$ state and are transferred to the target $\left|f=2, m_{f}=-2\right\rangle$ state by rapid adiabatic passage. $f$ is the total angular momentum of the atom and $m_{f}$ is its projection. The transfer is based on a 1-ms radio frequency sweep performed at an external magnetic field of about 199 G. At this magnetic field, losses are small in both the initial and final states and during the transfer. The sample contains up to $3 \times 10^{5}$ atoms at $650 \mathrm{nK}$ in a trap with an average frequency of $2 \pi \times 114(5) \mathrm{Hz}$.

To locate the $d$-wave resonances, we observe that the atom number decreased due to inelastic two-body losses in the proximity of the Feshbach resonance. We ramp the magnetic field strength in $10 \mathrm{~ms}$ to the target value. After a fixed holding time, chosen to not lead to complete depletion of the atoms at resonance, the magnetic field is ramped back to the magnetic field strength where high-field absorption imaging of the remaining atoms is performed [17]. Figure 1 shows the remaining atom fraction at different values of the magnetic field strength in the vicinity of the predicted $d$-wave Feshbach resonances. By fitting the loss data with phenomenological Gaussian curves, we obtain the following three resonance positions: 125.94(14), 188.72(5), and 227.71(60) G. The predicted width of the resonance at $188.72 \mathrm{G}$ is far below our magnetic field stability of about $30 \mathrm{mG}$ and leads to experimental points not following a Gaussian profile; see Fig. 1(b).

We also measure the remaining atom number at the resonance positions for variable holding time. The data are shown in Figs. 1(d)-1(f) for the 125.94, 188.72, and 227.71 G resonances, respectively. The inelastic loss rate coefficients are obtained from a fit to the data according to the two-body loss differential equation including the effects of antievaporation heating $[18,19]$ and background lifetime (about $17 \mathrm{~s}$ ). The loss rate coefficients are summarized in Fig. 1(g) and confirm the expected large difference between fast [Fig. 1(e)] and slow [Figs. 1(d) and 1(f)] losses despite the same $d$-wave character 

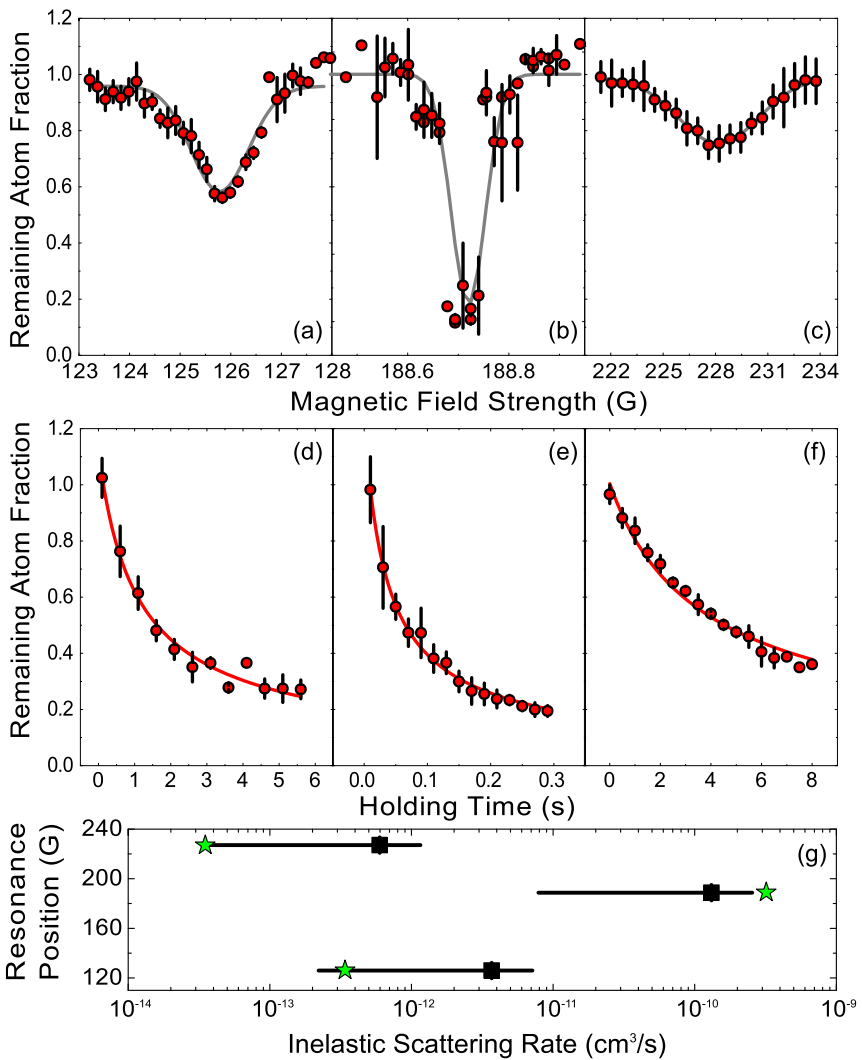

FIG. $1 .{ }^{39} \mathrm{~K} d$-wave Feshbach resonances in the hyperfine state $\left|f=2, m_{f}=-2\right\rangle$. [(a)-(c)] The remaining fraction of ${ }^{39} \mathrm{~K}$ atoms is measured for different values of the magnetic field. The holding times for the three measurements are $1 \mathrm{~s}, 470 \mathrm{~ms}$, and $4 \mathrm{~s}$, respectively. Gray lines are the phenomenological Gaussian fit curves. Error bars are the standard deviation of different experimental runs. [(d)-(f)] Remaining atom fraction as a function of time for the $d$-wave Feshbach resonances in panels (a), (b), and (c), respectively. The measurements are performed at the magnetic fields where losses are maximal. The lines are fits according to the two-body loss rate equation; see text. (g) Comparison between the measured (black squares) and predicted (green stars) inelastic loss rates. For the uncertainties on the loss rate, see text.

of the resonances. The values are in good agreement with theoretical predictions using the results of Sec. IV within the error bars, which include statistical uncertainties and the uncertainties on the calibration of temperature, trap frequency, and atom number. The values for the $125.94 \mathrm{G}$ [Figs. 1(a) and 1(d)] and $227.71 \mathrm{G}$ [Figs. 1(c) and 1(f)] resonances are larger than predicted, probably because of other loss contributions not included in the fit. The measured value for the $188.7 \mathrm{G}$ [Figs. 1(b) and 1(e)] resonance is instead smaller than expected as the narrow resonance width and the magnetic field jitter do not allow to remain exactly at resonance.

The resonance measurements were only performed for one sample temperature. We checked with the derived potential system; see Sec. IV for the temperature dependence of the resonance positions. Changing the kinetic energy from 1 to $0.5 \mu \mathrm{K}$ results in a shift of the positions by less than $5 \mathrm{mG}$, which is much smaller than the present experimental uncertainty.

\section{ANALYSIS}

We start our analysis from potential functions of the two lowest electronic states $X^{1} \Sigma_{o}^{+}$and $a^{3} \Sigma_{u}^{+}$derived from spectroscopic observation which have been described in detail in [7]. We refit the spectroscopic data with a smaller set of potential parameters to reduce the risk of obtaining unphysical tiny oscillatory behavior of the potential function. In Ref. [7], state $X^{1} \Sigma_{g}^{+}$was described by 31 parameters, but now 22 are sufficient. For state $a^{3} \Sigma_{u}^{+}$, we use 14 parameters, compared to 22 in the previous work. The resulting potentials form the starting point for a fit of 49 Feshbach resonances and a comparison of the experimentally determined Feshbach resonance positions with the ones resulting from the coupled channel calculation. We identify the Feshbach resonance position by the maximum scattering rate coefficient at the kinetic energy given by the experimental conditions. We base our analysis on Feshbach resonance data for the isotope combinations ${ }^{39} \mathrm{~K}-{ }^{39} \mathrm{~K}$ from D'Errico et al. [11] and Refs. [14,20], ${ }^{40} \mathrm{~K}-{ }^{40} \mathrm{~K}$ from Regal et al. $[21,23],{ }^{39} \mathrm{~K}-{ }^{41} \mathrm{~K}$ from Tanzi et al. $[14],{ }^{41} \mathrm{~K}{ }^{-41} \mathrm{~K}$ from Chen et al. [13] and Tanzi et al. [14], and ${ }^{40} \mathrm{~K}-{ }^{41} \mathrm{~K}$ from $\mathrm{Wu}$ et al. [12]. We summarize the data in Table I with their quantum numbers and the reported experimental uncertainty. As quantum numbers, we use the projection $M$ of the total angular momentum onto the field axis, the atom pair labels for dressed states, and the interval $l_{\min }-l_{\max }$ of the partial waves. The labels of the atomic dressed states are given by $\left|f, m_{f}\right\rangle$. The column "type" indicates that for "el" the peak of the elastic part of the rate coefficient is taken and for "in" the sum of the inelastic contributions.

The evaluation uses atomic hyperfine and $g$ factors from Ref. [24]. We fit the data in Table I to the BO potentials, adjusting the branches at small $\left(R<R_{\text {in }}\right)$ and large $\left(R>R_{\text {out }}\right)$ internuclear separations. After a few trials, it became clear that the three data points for the ${ }^{40} \mathrm{~K}-{ }^{41} \mathrm{~K}$ isotope combination show significant deviations (several times the experimental uncertainty) compared to all other isotope combinations. In the following, we thus exclude these resonances from the analysis and report only their resulting deviations in the final conclusion [25]. Refitting all remaining resonances, we obtain a normalized standard deviation of $\sigma=0.977$. These results are given in Table I in column "o-c(1)" (observed-calculated) and in row 1 of Table II [labeled model (1)]. Analyzing the obtained fit for the different isotope combinations [see Table II, model (1)] reveals that the main part of the sum of squared weighted deviations stems from the isotope combination ${ }^{39} \mathrm{~K}-{ }^{41} \mathrm{~K}$, resulting in $\sigma=1.235$, whereas the other isotope combinations show values below 0.72 . A separate fit to the data of the isotope combination ${ }^{39} \mathrm{~K}-{ }^{41} \mathrm{~K}$ only emphasizes the consistency of these observations with a resulting normalized standard deviation of $\sigma=0.753$ [see Table II, model $\left.\left({ }^{39} \mathrm{~K}^{41} \mathrm{~K}\right)_{a}\right]$. We thus started an additional fit in an attempt to optimize the result for the isotope combination ${ }^{39} \mathrm{~K}-{ }^{41} \mathrm{~K}$ by applying the potentials from the separate fit of ${ }^{39} \mathrm{~K}-{ }^{41} \mathrm{~K}$ above as initial values. Note that nonlinear fits regularly give slightly different results depending on initial values since it is hard to find the global minimum of the sum of squared weighted deviations. In this second fit, we obtain almost the same overall fit quality with a normalized standard deviation of $\sigma=0.993$ but now the main deviation 
TABLE I. Feshbach resonances and their theoretical modeling. Column o-c gives the difference of observed field value [obs] and experimental uncertainty [unc)] to calculated one (not listed). The number indicates the model of evaluation as in Table II: (1) pure $\mathrm{BO}$ potentials, (2) adding $\mathrm{BO}$ correction $\mathrm{BO}_{\text {gen }}$ for the general case, and (3) adding $\mathrm{BO}$ corrections $\mathrm{BO}_{\text {gen }}$ and $\mathrm{BO}_{\text {asym }}$ for the homo- and heteronuclear cases.

\begin{tabular}{|c|c|c|c|c|c|c|c|c|c|c|c|}
\hline Isotope & $M$ & Atom pair & $l_{\min }$ & $l_{\max }$ & Type & Obs $[\mathrm{G}]$ & Unc $[\mathrm{G}]$ & $\mathrm{o}-\mathrm{c}(1)[\mathrm{G}]$ & $\mathrm{o}-\mathrm{c}(2)[\mathrm{G}]$ & o-c(3) $[\mathrm{G}]$ & Ref. \\
\hline \multirow[t]{13}{*}{$39 / 39$} & 2 & $|1,1\rangle|1,1\rangle$ & 0 & 0 & el & 403.4 & 0.7 & 0.958 & 0.876 & 0.849 & [11] \\
\hline & 2 & $|1,1\rangle|1,1\rangle$ & 0 & 0 & el & 752.3 & 0.1 & 0.104 & 0.024 & 0.039 & \\
\hline & 2 & $|1,1\rangle|1,1\rangle$ & 0 & 0 & el & 25.85 & 0.1 & 0.016 & -0.033 & -0.024 & \\
\hline & 0 & $|1,0\rangle|1,0\rangle$ & 0 & $2^{\mathrm{a}}$ & el & 59.3 & 0.6 & 0.400 & 0.315 & 0.320 & \\
\hline & 0 & $|1,0\rangle|1,0\rangle$ & 0 & 0 & el & 66.0 & 0.9 & 0.491 & 0.421 & 0.430 & \\
\hline & -2 & $|1,-1\rangle|1,-1\rangle$ & 0 & 0 & el & 32.6 & 1.5 & -1.006 & -0.979 & -0.982 & \\
\hline & -2 & $|1,-1\rangle|1,-1\rangle$ & 0 & 0 & el & 162.8 & 0.9 & 0.503 & 0.460 & 0.467 & \\
\hline & -2 & $|1,-1\rangle|1,-1\rangle$ & 0 & 0 & el & 562.2 & 1.5 & 1.383 & 1.301 & 1.274 & \\
\hline & -2 & $|1,-1\rangle|1,-1\rangle$ & 0 & 2 & in & 395.1 & 1.0 & -0.020 & -0.122 & -0.160 & {$[20]$} \\
\hline & -1 & $|1,0\rangle|1,-1\rangle$ & 0 & 0 & el & 113.76 & 0.1 & 0.062 & -0.002 & 0.007 & {$[14]$} \\
\hline & -4 & $|2,-2\rangle|2,-2\rangle$ & 0 & 2 & in & 125.94 & 0.14 & -0.097 & -0.125 & -0.120 & present \\
\hline & -4 & $|2,-2\rangle|2,-2\rangle$ & 0 & 2 & in & 188.72 & 0.05 & 0.033 & -0.008 & 0.000 & \\
\hline & -4 & $|2,-2\rangle|2,-2\rangle$ & 0 & 2 & in & 227.71 & 0.60 & -0.150 & -0.170 & -0.130 & \\
\hline \multirow[t]{6}{*}{$40 / 40$} & -8 & $|9 / 2,-9 / 2\rangle|9 / 2,-7 / 2\rangle$ & 0 & 0 & el & 202.10 & 0.07 & 0.003 & -0.014 & 0.005 & [21] \\
\hline & -7 & $|9 / 2,-9 / 2\rangle|9 / 2,-5 / 2\rangle$ & 0 & 0 & el & 224.21 & 0.05 & -0.001 & -0.018 & 0.003 & \\
\hline & -6 & $|9 / 2,-7 / 2\rangle|9 / 2,-5 / 2\rangle$ & 0 & 0 & el & 174.0 & 2.0 & -0.280 & -0.155 & -0.130 & \\
\hline & -7 & $|9 / 2,-7 / 2\rangle|9 / 2,-7 / 2\rangle$ & 1 & 1 & el & 198.81 & 0.05 & -0.010 & -0.044 & -0.024 & [22] \\
\hline & -8 & $|9 / 2,-7 / 2\rangle|9 / 2,-7 / 2\rangle$ & 1 & 1 & el & 198.34 & 0.05 & 0.030 & -0.006 & 0.014 & \\
\hline & 7 & $|9 / 2,7 / 2\rangle|9 / 2,7 / 2\rangle$ & 1 & 1 & el & 436.3 & 0.5 & -0.484 & -0.444 & -0.440 & [21] \\
\hline \multirow[t]{23}{*}{$39 / 41$} & -1 & $|1,-0\rangle|1,-1\rangle$ & 0 & 0 & el & 228.88 & 0.08 & -0.091 & -0.036 & -0.035 & {$[14]$} \\
\hline & -2 & $|1,-1\rangle|1,-1\rangle$ & 0 & 0 & el & 149.84 & 0.06 & 0.100 & 0.083 & 0.082 & \\
\hline & -3 & $|1,-1\rangle|2,-2\rangle$ & 0 & 0 & el & 649.6 & 0.6 & 0.564 & 0.535 & 0.343 & \\
\hline & 3 & $|1,1\rangle|2,2\rangle$ & 0 & 0 & el & 341.5 & 0.20 & -0.018 & 0.097 & 0.101 & \\
\hline & 3 & $|1,1\rangle|2,2\rangle$ & 0 & 0 & el & 353.8 & 0.20 & -0.092 & 0.017 & 0.023 & \\
\hline & 2 & $|1,1\rangle|1,1\rangle$ & 0 & 0 & el & 139.27 & 0.04 & -0.072 & -0.020 & -0.016 & \\
\hline & 2 & $|1,1\rangle|1,1\rangle$ & 0 & 0 & el & 146.24 & 0.07 & -0.109 & -0.057 & -0.056 & \\
\hline & 2 & $|1,1\rangle|1,1\rangle$ & 0 & 0 & el & 338.12 & 0.07 & -0.057 & 0.032 & 0.024 & \\
\hline & 2 & $|1,1\rangle|1,1\rangle$ & 0 & 0 & el & 500.2 & 0.3 & 0.276 & 0.242 & 0.051 & \\
\hline & 2 & $|1,1\rangle|1,1\rangle$ & 0 & 0 & el & 518.4 & 0.1 & 0.072 & 0.049 & -0.124 & \\
\hline & 1 & $|1,1\rangle|1,0\rangle$ & 0 & 0 & el & 88.2 & 0.1 & -0.212 & -0.157 & -0.160 & \\
\hline & 1 & $|1,1\rangle|1,0\rangle$ & 0 & 0 & el & 160.05 & 0.06 & -0.020 & 0.034 & 0.037 & \\
\hline & 1 & $|1,1\rangle|1,0\rangle$ & 0 & 0 & el & 165.80 & 0.05 & -0.069 & -0.016 & -0.015 & \\
\hline & 1 & $|1,1\rangle|1,0\rangle$ & 0 & 0 & el & 344.4 & 0.1 & -0.006 & 0.073 & 0.053 & \\
\hline & 1 & $|1,1\rangle|1,0\rangle$ & 0 & 0 & el & 522.6 & 0.2 & 0.246 & 0.217 & 0.028 & \\
\hline & 1 & $|1,1\rangle|1,0\rangle$ & 0 & 0 & el & 553.1 & 0.1 & 0.243 & 0.229 & 0.069 & \\
\hline & 0 & $|1,1\rangle|1,-1\rangle$ & 0 & 0 & el & 189.88 & 0.05 & -0.053 & 0.000 & 0.001 & \\
\hline & 0 & $|1,1\rangle|1,-1\rangle$ & 0 & 0 & el & 348.4 & 0.1 & 0.040 & 0.110 & 0.076 & \\
\hline & 0 & $|1,1\rangle|1,-1\rangle$ & 0 & 0 & el & 384.91 & 0.07 & -0.044 & 0.049 & 0.049 & \\
\hline & 0 & $|1,1\rangle|1,-1\rangle$ & 0 & 0 & el & 553.5 & 0.2 & 0.249 & 0.223 & 0.044 & \\
\hline & -1 & $|1,0\rangle|1,-1\rangle$ & 0 & 0 & el & 228.88 & 0.08 & -0.091 & -0.036 & -0.035 & \\
\hline & -2 & $|1,-1\rangle|1,-1\rangle$ & 0 & 0 & el & 149.84 & 0.06 & 0.100 & 0.083 & 0.082 & \\
\hline & -3 & $|1,-1\rangle|2,-2\rangle$ & 0 & 0 & el & 649.6 & 0.6 & 0.564 & 0.535 & 0.343 & \\
\hline \multirow[t]{4}{*}{$41 / 41$} & -2 & $|1,-1\rangle|1,-1\rangle$ & 0 & 0 & el & 51.1 & 0.2 & -0.021 & -0.100 & -0.101 & {$[14]$} \\
\hline & -1 & $|1,-1\rangle|1,0\rangle$ & 0 & 0 & el & 51.92 & 0.08 & 0.022 & -0.077 & -0.081 & \\
\hline & 2 & $|1,1\rangle|1,1\rangle$ & 0 & 0 & el & 409.2 & 0.2 & -0.160 & 0.053 & 0.068 & [23] \\
\hline & 2 & $|1,1\rangle|1,1\rangle$ & 0 & 0 & el & 660.6 & 0.2 & -0.015 & 0.000 & 0.059 & \\
\hline \multirow[t]{3}{*}{$40 / 41$} & $11 / 2$ & $|9 / 2,9 / 2\rangle|1,1\rangle$ & 0 & 2 & el & 472.6 & 0.3 & -2.150 & -1.922 & -1.906 & [12] \\
\hline & $11 / 2$ & $|9 / 2,9 / 2\rangle|1,1\rangle$ & 1 & 1 & el & 432.9 & 0.3 & -2.651 & -2.280 & -2.252 & \\
\hline & $11 / 2$ & $|9 / 2,9 / 2\rangle|1,1\rangle$ & 0 & 0 & el & 542.7 & 1.0 & 0.167 & 0.402 & 0.416 & \\
\hline
\end{tabular}

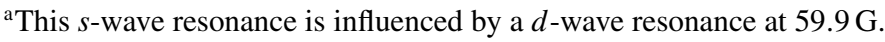

lies in the ${ }^{40} \mathrm{~K}-{ }^{40} \mathrm{~K}$ isotope combination with its individual value $\sigma=1.566$ as given in the third line of Table II, model $\left({ }^{39} \mathrm{~K}^{41} \mathrm{~K}\right)_{b}$. Since the reduced masses of ${ }^{39} \mathrm{~K}{ }^{41} \mathrm{~K}$ and ${ }^{40} \mathrm{~K}-{ }^{40} \mathrm{~K}$ are almost equal, the different behavior of these two isotope combinations in the two different fits [model (1) and model $\left({ }^{39} \mathrm{~K}^{41} \mathrm{~K}\right)_{b}$ ] give a strong hint that mass effects beyond the simple mass scaling of the rovibrational motion should be considered. 
TABLE II. Overview of obtained standard deviations at different evaluation steps. The column "model" gives the same numbers as in Table I. Columns labeled by isotope combinations show the contribution of that combination: (1) BOA; $(39 / 41)_{a}$, fit restricted to ${ }^{39} \mathrm{~K}-{ }^{41} \mathrm{~K}$; (39/41) , as (1) using (39/41) as starting guess; (2), BOA with $\mathrm{BO}_{\text {gen }}$ corrections; (3), $\mathrm{BOA}$ with $\mathrm{BO}_{\text {gen }}$ and $\mathrm{BO}_{\text {asym }}$ corrections.

\begin{tabular}{lccccc}
\hline \hline & \multicolumn{5}{c}{$\sigma$} \\
\cline { 2 - 6 } Model & Total & ${ }^{39} \mathrm{~K}-{ }^{41} \mathrm{~K}$ & ${ }^{40} \mathrm{~K}-{ }^{40} \mathrm{~K}$ & ${ }^{39} \mathrm{~K}-{ }^{39} \mathrm{~K}$ & ${ }^{41} \mathrm{~K}-{ }^{41} \mathrm{~K}$ \\
\hline$(1)$ & 0.977 & 1.235 & 0.476 & 0.718 & 0.428 \\
$(39 / 41)_{\mathrm{a}}$ & & 0.753 & & & \\
$(39 / 41)_{\mathrm{b}}$ & 0.993 & 0.867 & 1.566 & 0.991 & 0.394 \\
$(2)$ & 0.786 & 0.952 & 0.541 & 0.593 & 0.556 \\
$(3)$ & 0.651 & 0.737 & 0.428 & 0.584 & 0.607 \\
\hline \hline
\end{tabular}

In the next step, we thus include beyond $\mathrm{BO}$ corrections proportional to the reduced mass for the general case [i.e., part $U_{\text {gen }}$ from Eq. (6)]. Because we can only study the small variations between the naturally existing isotope combinations, it is advantageous to define one isotope combination as a reference. This results in a parametrization of the full potentials with $\mathrm{BO}$ corrections for molecule $A B$ given by

$$
\begin{aligned}
U(R)= & U_{\mathrm{BO}}(R)+\mathrm{BO}_{\mathrm{gen}}(R)\left(1-\frac{\mu_{\mathrm{ref}}}{\mu_{A B}}\right) \\
& +\mathrm{BO}_{\text {asym }}(R)\left(\frac{M_{A}-M_{B}}{M_{A}+M_{B}}\right)^{2},
\end{aligned}
$$

where the factor of the electron mass in Eq. (6) is incorporated in the new functions $\mathrm{BO}_{\text {gen }}$ and $\mathrm{BO}_{\text {asym }}$ and $\mu_{\text {ref }}$ is the reduced mass of the reference combination. Here we apply ${ }^{39} \mathrm{~K}-{ }^{39} \mathrm{~K}$ as a reference. For this combination, we have a large number of $s$-wave resonances and additionally also $d$-wave resonances. Both together fix the asymptotic branch of the potentials. This is different for the ${ }^{39} \mathrm{~K}-{ }^{41} \mathrm{~K}$ isotope combination where only $s$-wave resonances have been measured. In principle, $\mathrm{BO}_{\text {gen }}$ is a function of $R$, but the present data set is too small to derive such function from a fit with acceptable significance. Thus, we simplify the condition by assuming correction functions to be proportional to the $\mathrm{BO}$ potential and Eq. (7) reads now

$$
\begin{aligned}
U(R)= & U_{\mathrm{BO}}(R)\left[1+\mathrm{BO}_{\mathrm{gen}}\left(1-\frac{\mu_{\mathrm{ref}}}{\mu_{A B}}\right)\right. \\
& \left.+\mathrm{BO}_{\text {asym }}\left(\frac{M_{A}-M_{B}}{M_{A}+M_{B}}\right)^{2}\right],
\end{aligned}
$$

where now $\mathrm{BO}_{\text {gen }}$ and $\mathrm{BO}_{\text {asym }}$ are fit parameters for the amplitude of the BO corrections. A crude justification of this assumption is that the normal mass effect in atomic physics, e.g., the Rydberg constant and its nuclear mass dependence, show a similar form of the correction for the binding energy. Furthermore, a molecular potential describes the variation of the kinetic energy within the nuclear vibrational motion as function of $R$ and is therefore a measure of the coupling to the electron motion.

Starting with $\mathrm{BO}_{\text {gen }}$ for states $X^{1} \Sigma_{g}^{+}$and $a^{3} \Sigma_{u}^{+}$we perform a fit of all resonances, adding the parameter for both electronic
TABLE III. Born-Oppenheimer corrections, according to Eq. (8). The number indicates the model of evaluation: (2) adding BO correction $\mathrm{BO}_{\text {gen }}$ for the general case and (3) adding $\mathrm{BO}$ corrections $\mathrm{BO}_{\text {gen }}$ and $\mathrm{BO}_{\text {asym }}$ for the general and heteronuclear cases. Values in brackets are not significantly determined and effectively zero.

\begin{tabular}{lcc}
\hline \hline Parameter & $X^{1} \Sigma_{g}^{+}$ & $a^{3} \Sigma_{u}^{+}$ \\
\hline $\mathrm{BO}_{\text {gen }}(2)$ & $(-0.00003)$ & -0.00046 \\
$\mathrm{BO}_{\text {gen }}(3)$ & $(-0.00006)$ & -0.00046 \\
$\mathrm{BO}_{\text {asym }}(3)$ & 0.0057 & $(-0.000001)$ \\
\hline
\end{tabular}

states, and obtain a normalized standard deviation of $\sigma=$ 0.786 (the individual deviations are shown in column "o-c(2)" in Table I). This value should be compared with the one from a fit of the pure BO potentials $\sigma=0.977$. Including beyond Born-Oppenheimer corrections apparently leads to a significantly better fit.

Looking again at details of the fit for the different isotope combinations in Table II model (2), we see that the combination ${ }^{39} \mathrm{~K}-{ }^{41} \mathrm{~K}$ is described with $\sigma=0.952$ whereas the other three show values below 0.6. Since we removed the isotope combination ${ }^{40} \mathrm{~K}-{ }^{41} \mathrm{~K}$ from the evaluation already earlier for another reason (see also Ref. [26]), the former one is the only heteronuclear case remaining for which the standard deviation is significantly larger than the value seen in a separate fit (comp. above 0.753). Keeping in mind that the isotope combination with almost equal reduced mass, namely ${ }^{40} \mathrm{~K}-{ }^{40} \mathrm{~K}$, is well represented by the introduced $\mathrm{BO}$ correction, we complement our model by the heteronuclear extension of the BO correction, which is already contained in Eq. (8) by the parameter $\mathrm{BO}_{\mathrm{asym}}$. The new fit results now in a standard deviation of $\sigma=0.651$ [see Table II model (3)] and thus a further improvement compared to 0.786 from model (2). Additionally, all individual standard deviations are almost equal to the values obtained by separated fits. The deviations of observation to calculation from the new fit are shown in column "o-c(3)" of Table I. The sequential improvement of the fit quality including beyond Born-Oppenheimer terms underlines the significance of corrections beyond Born-Oppenheimer for the precise description of molecular potentials and the precision derivation of atomic scattering properties [27].

In Table III, we give the magnitude of the BO corrections for the two electronic states $X^{1} \Sigma_{g}^{+}$and $a^{3} \Sigma_{u}^{+}$. The uncertainty of the significantly determined parameters is about $20 \%$. For better insight into the $\mathrm{BO}$ correction, we calculate the highest vibrational levels with the correction and compare them with the level energy, setting the correction to zero. For the heaviest isotope ${ }^{41} \mathrm{~K}-{ }^{41} \mathrm{~K}$ and thus the largest difference to the reference isotope ${ }^{39} \mathrm{~K}-{ }^{39} \mathrm{~K}$, we obtain for the level $v=27, N=0$ of the state $a^{3} \Sigma_{u}^{+}$a difference of $220 \mathrm{kHz}$ and for the state $X^{1} \Sigma_{g}^{+}(v=87, N=0)$ it is effectively zero, because here the influence by BO correction appears only for heteronuclear isotope combinations.

\section{DISCUSSION}

From the different models, we calculated the scattering lengths for the pure singlet and triplet states. The results are 
TABLE IV. Scattering lengths in units of $a_{0}$ of all natural isotope combinations of $\mathrm{K}$ derived from the different potential models.

\begin{tabular}{lrrcc}
\hline \hline Isotope & (1) $X^{1} \Sigma_{g}^{+}$ & (3) $X^{1} \Sigma_{g}^{+}$ & (1) $a^{3} \Sigma_{u}^{+}$ & (3) $a^{3} \Sigma_{u}^{+}$ \\
\hline $39 / 39$ & 138.801 & $138.759(20)$ & -33.376 & $-33.413(25)$ \\
$39 / 40$ & -2.669 & $-2.707(15)$ & -2031 & $-2026(10)$ \\
$39 / 41$ & 113.094 & $113.036(20)$ & 176.600 & $176.688(25)$ \\
$40 / 40$ & 104.416 & $104.410(20)$ & 169.204 & $169.288(25)$ \\
$40 / 41$ & -54.447 & $-54.479(25)$ & 97.139 & $97.186(20)$ \\
$41 / 41$ & 85.400 & $85.409(18)$ & 60.266 & $60.317(18)$ \\
\hline \hline
\end{tabular}

summarized in Table IV for the different isotope combinations using model (1) (BO approximation) and (3) (including all beyond $\mathrm{BO}$ corrections). Because we choose the isotope combination ${ }^{39} \mathrm{~K}-{ }^{39} \mathrm{~K}$ as reference, one might expect no difference for the resulting scattering lengths for this isotope pair when using model (1) or (3) respectively. However, we do observe corrections (see Table IV). Equal values for ${ }^{39} \mathrm{~K}-{ }^{39} \mathrm{~K}$ would result if the evaluation in case (3) would only vary the BO-correction parameters and anything else would be kept constant. This will not be the optimal fit strategy, because in case (1), i.e., no BO corrections, existing significant BO corrections are distributed over the deviations of the fit over all isotopes and thus also the reference isotope is influenced. One can see such different distribution from the standard deviations of ${ }^{39} \mathrm{~K}-{ }^{39} \mathrm{~K}$ given for models (1) and (3) in Table II, the former one is larger than the latter one. Because of this influence, we only give error estimates for the complete model including BO corrections in Table IV and the differences between models (1) and (3) do not show the true magnitude of the BO correction. See also the calculation of the energy shift by the $\mathrm{BO}$ correction as given at the end of Sec. IV.

A complete list of scattering lengths was reported in Ref. [7]. The new values show a significant improvement by roughly a factor 5 of the error limit. The values agree in most cases within uncertainty limits despite the fact that the former evaluation could only incorporate Feshbach resonances for ${ }^{39} \mathrm{~K}-{ }^{39} \mathrm{~K}$ and ${ }^{40} \mathrm{~K}-{ }^{40} \mathrm{~K}$. The paper stated that a weak indication of $\mathrm{BO}$ corrections could be obtained from the resonances. We believe the present evaluation shows this clearly. Additionally, we were able to study the difference between the homonuclear and heteronuclear cases, resulting in the values of $\mathrm{BO}_{\text {gen }}$ and $\mathrm{BO}_{\text {asym }}$.

We obtained a significant contribution for the triplet state $a^{3} \Sigma_{u}^{+}$by $\mathrm{BO}_{\text {gen }}$ for both fit cases but for the singlet state $X^{1} \Sigma_{g}^{+}$only for the heteronuclear isotope pairs. This is probably related to the fact that the closest singlet state, namely $A^{1} \Sigma_{u}^{+}$, has $u$ symmetry compared to $g$ symmetry for the singlet ground state. These two can only couple by the symmetry breaking part of the Hamiltonian responsible for the BO correction [28]. The situation for the triplet state is different, where the energetically closest is $b^{3} \Pi_{u}$ and has $u$ symmetry as the triplet ground state. We should note that the magnitudes of both effects, $\mathrm{BO}_{\text {gen }}$ and $\mathrm{BO}_{\text {asym }}$ cannot be directly compared, because the former one is referenced to ${ }^{39} \mathrm{~K}-{ }^{39} \mathrm{~K}$ and thus describes only the difference between the isotope pairs whereas the latter indicates the total effect.

We evaluated the isotope dependence by using the precise Feshbach spectroscopy and checked finally that the obtained
BO corrections have little influence in the deep rovibrational levels measured by molecular spectroscopy, e.g., in Refs. $[7,29,30]$, which have an uncertainty in the order of few thousands of $\mathrm{cm}^{-1}$ or about $100 \mathrm{MHz}$ compared to $1 \mathrm{MHz}$ or better for the Feshbach spectroscopy. For this purpose, we went back to the full data set from the spectroscopy for iterating the fit for obtaining the consistent description of the complete data set from molecular and Feshbach spectroscopy. The final parameter sets of the potentials are given in the Appendix; see Tables V-VII.

\section{CONCLUSION AND OUTLOOK}

We use an analysis of the complete set of all known Feshbach resonances in different $\mathrm{K}$ isotope combinations to derive potential energy curves for states $X^{1} \Sigma_{g}^{+}$and $a^{3} \Sigma_{u}^{+}$ and find clear signatures of beyond $\mathrm{BO}$ corrections. We base our work on the discussion of $\mathrm{H}_{2}$ and $\mathrm{HD}$ molecules by van Vleck [15] and find correction terms for the homonuclear and heteronuclear cases when analyzing homo- and heteronuclear isotope combinations of $\mathrm{K}$ respectively. Unfortunately, our analysis of heteronuclear cases is restricted to the ${ }^{39} \mathrm{~K}-{ }^{41} \mathrm{~K}$ isotope combination, although, in principle, more isotope combinations exist. However, available Feshbach resonance data of the ${ }^{40} \mathrm{~K}^{-}{ }^{41} \mathrm{~K}$ [12] isotope combination show very large deviations which are beyond a realistic description [25]. We therefore excluded this isotope combination from the analysis given in Sec. IV. To allow for an extended analysis of heteronuclear beyond BO corrections, it would be very much desirable to revisit observed Feshbach resonances in the $|9 / 2,9 / 2\rangle+|1,1\rangle$ channel of the ${ }^{40} \mathrm{~K}-{ }^{-11} \mathrm{~K}$ isotope combination and extend measurements to resonances within other collision channels such as $|9 / 2,-9 / 2\rangle+|1,1\rangle$. In the same context, the ${ }^{39} \mathrm{~K}-{ }^{40} \mathrm{~K}$ isotope is of great interest. Here, it would be particularly favorable to study collisions in the $|9 / 2,-7 / 2\rangle+$ $|1,1\rangle$ and $|9 / 2,-5 / 2\rangle+|1,1\rangle$ channels. In these channels, well-separated Feshbach resonances in a magnetic field region below $200 \mathrm{G}$ should be found, whereas sharp resonances in the $|9 / 2,-9 / 2\rangle+|1,1\rangle$ channel will be overlapped by a very broad resonance. Furthermore, the above-mentioned channels will show sharp Feshbach resonances in the range of 800 to $850 \mathrm{G}$. We believe that such studies will settle the discussion of the importance of BO corrections in cases of homo- and heteronuclear pairs of homopolar molecules.

In the same spirit, it would be very interesting to analyze Feshbach resonances in the different isotope combinations of the homopolar molecule $\mathrm{Li}_{2}$. There exists a detailed analysis [31] of spectroscopic data of the $X^{1} \Sigma_{g}^{+}-A^{1} \Sigma_{u}^{+}$transition in the $\mathrm{Li}_{2}$ considering homonuclear $\mathrm{BO}$ corrections $\left[\mathrm{BO}_{\text {gen }}(R)\right.$ from Eq. (7)]. The study includes data from ${ }^{7} \mathrm{Li}-{ }^{6} \mathrm{Li}$ isotopologue; however, the authors do not mention any need to distinguish between homo- and heteronuclear corrections. The data set for the ${ }^{7} \mathrm{Li}-{ }^{6} \mathrm{Li}$ molecule is small compared to that of both homonuclear molecules ${ }^{7} \mathrm{Li}-{ }^{7} \mathrm{Li}$ and ${ }^{6} \mathrm{Li}-{ }^{6} \mathrm{Li}$, and thus it could be not sufficiently significant for the above mentioned distinction. For $\mathrm{Li}_{2}$ there exist also measurements of Feshbach resonances for the homonuclear cases (see the latest report by Gerken et al. [32]), but nothing on ${ }^{7} \mathrm{Li}-{ }^{6} \mathrm{Li}$. Thus, studies of Feshbach resonances of $\mathrm{Li}-\mathrm{Li}$ would be worthwhile 
TABLE V. Parameters of the analytic representation of the $X^{1} \Sigma_{g}^{+}$state potential with adiabatic Born-Oppenheimer correction and reference isotopologue ${ }^{39} \mathrm{~K}-{ }^{39} \mathrm{~K}$. The energy reference is the dissociation asymptote. Parameters with ${ }^{*}$ are set for continuous extrapolation of the potential.

\begin{tabular}{lc}
\hline \hline & $R<R_{\text {in }}=2.87 \AA$ \\
\hline$A^{*}$ & $-0.2600158561 \times 10^{4} \mathrm{~cm}^{-1}$ \\
$B^{*}$ & $0.8053173040 \times 10^{9} \mathrm{~cm}^{-1} \AA^{12}$ \\
$N_{s}$ & 12 \\
& $R_{\text {in }} \leqslant R \leqslant R_{\text {out }}=12.000 \AA$ \\
$b$ & -0.39 \\
$R_{\mathrm{m}}$ & $3.9243617 \AA$ \\
$a_{0}$ & $-4450.9007703 \mathrm{~cm}^{-1}$ \\
$a_{1}$ & $0.159877863995326747 \mathrm{~cm}^{-1}$ \\
$a_{2}$ & $0.141337574101676037 \times 10^{5} \mathrm{~cm}^{-1}$ \\
$a_{3}$ & $0.107669620493846905 \times 10^{5} \mathrm{~cm}^{-1}$ \\
$a_{4}$ & $-0.331314023322698995 \times 10^{4} \mathrm{~cm}^{-1}$ \\
$a_{5}$ & $-0.163943210499613087 \times 10^{5} \mathrm{~cm}^{-1}$ \\
$a_{6}$ & $-0.216334200177141829 \times 10^{5} \mathrm{~cm}^{-1}$ \\
$a_{7}$ & $-0.384655804768731250 \times 10^{5} \mathrm{~cm}^{-1}$ \\
$a_{8}$ & $-0.768229889574501722 \times 10^{5} \mathrm{~cm}^{-1}$ \\
$a_{9}$ & $0.157896664088991121 \times 10^{6} \mathrm{~cm}^{-1}$ \\
$a_{10}$ & $0.833691806464401074 \times 10^{6} \mathrm{~cm}^{-1}$ \\
$a_{11}$ & $-0.115890452663354226 \times 10^{7} \mathrm{~cm}^{-1}$ \\
$a_{12}$ & $-0.653607110081680864 \times 10^{7} \mathrm{~cm}^{-1}$ \\
$a_{13}$ & $0.487172809603480622 \times 10^{7} \mathrm{~cm}^{-1}$ \\
$a_{14}$ & $0.308101362964722812 \times 10^{8} \mathrm{~cm}^{-1}$ \\
$a_{15}$ & $-0.863340173933527432 \times 10^{7} \mathrm{~cm}^{-1}$ \\
$a_{16}$ & $-0.811804637748816609 \times 10^{8} \mathrm{~cm}^{-1}$ \\
$a_{17}$ & $0.492251670364311151 \times 10^{7} \mathrm{~cm}^{-1}$ \\
$a_{18}$ & $0.121156746090629265 \times 10^{9} \mathrm{~cm}^{-1}$ \\
$a_{19}$ & $0.280059277888290165 \times 10^{7} \mathrm{~cm}^{-1}$ \\
$a_{20}$ & $-0.968951931944736689 \times 10^{8} \mathrm{~cm}^{-1}$ \\
$a_{21}$ & $-0.314874358611015789 \times 10^{7} \mathrm{~cm}^{-1}$ \\
$a_{22}$ & $0.324661526246530302 \times 10^{8} \mathrm{~cm}^{-1}$ \\
$\mathrm{BO}_{\mathrm{gen}}$ & $(-0.00006)$ \\
$\mathrm{BO}_{\mathrm{asym}}$ & 0.00566 \\
\hline \hline & \\
\hline &
\end{tabular}

to investigate both homonuclear and heteronuclear beyond $\mathrm{BO}$ corrections.

We conclude that very interesting Feshbach spectroscopy is ahead of us to work out and highlight the importance of BO corrections in the understanding of cold collisions.

Recently, we became aware that in the doctoral thesis of Antje Ludewig, University Amsterdam [33], a large number of unpublished Feshbach resonances of ${ }^{40} \mathrm{~K}$ are reported and that in Liu et al. [34] $d$-wave resonances for ${ }^{41} \mathrm{~K}$ are reported. We checked these data with our model and found complete consistency for the data from Antje Ludewig after reassignment of some $p$-wave resonances. For the resonances in Liu et al., we find mainly consistency for the high-field data but the low-field data deviate systematically due to their strong temperature dependence, the analysis of which would require the inclusion of the kinetic energy dependence of the collision rates and the thermal distribution in the actual experiment. The description by a single-channel approximation for so-called "broad" resonances assumed by Liu et al. is not justified because the resonances show strong inelastic con-
TABLE VI. Parameters of the long-range part of the potentials for both states $X^{1} \Sigma_{g}^{+}$and $a^{3} \Sigma_{u}^{+}$.

\begin{tabular}{lc}
\hline \hline & $R_{\text {out }}<R$ \\
\hline$U_{\infty}$ & $0.0 \mathrm{~cm}^{-1}$ \\
$C_{6}$ & $0.1892338370 \times 10^{8} \mathrm{~cm}^{-1} \AA^{6}$ \\
$C_{8}$ & $0.5706799528 \times 10^{9} \mathrm{~cm}^{-1} \AA^{8}$ \\
$C_{10}$ & $0.1853042723 \times 10^{11} \mathrm{~cm}^{-1} \AA^{10}$ \\
$A_{\text {ex }}$ & $0.90092159 \times 10^{4} \mathrm{~cm}^{-1} \AA^{-\gamma}$ \\
$\gamma$ & 5.19500 \\
$\beta$ & $2.13539 \AA^{-1}$ \\
\hline \hline
\end{tabular}

tributions. Details of these calculations are contained in the Appendix.

\section{ACKNOWLEDGMENTS}

We gratefully acknowledge financial support from the European Research Council through ERC Starting Grant POLAR and from the Deutsche Forschungsgemeinschaft (DFG) through CRC 1227 (DQ-mat), Project No. A03, and FOR 2247, Project No. E5. K.K.V. and P.G. thank the Deutsche Forschungsgemeinschaft for financial support through $\mathrm{Re}$ search Training Group 1991.

TABLE VII. Parameters of the analytic representation of the $a^{3} \Sigma_{u}^{+}$state potential with adiabatic Born-Oppenheimer correction and reference isotopologue ${ }^{39} \mathrm{~K}_{2}$. The energy reference is the dissociation asymptote. Parameters with * are set for continuous extrapolation of the potential.

\begin{tabular}{lc}
\hline \hline \multicolumn{2}{c}{$R<R_{\text {in }}=4.755 \AA$} \\
\hline$A^{*}$ & $-0.7009379657 \times 10^{3} \mathrm{~cm}^{-1}$ \\
$B^{*}$ & $0.80690073665 \times 10^{7} \mathrm{~cm}^{-1} \AA^{6}$ \\
$N_{s}$ & 6 \\
& $R_{\text {in }} \leqslant R \leqslant R_{\text {out }}=12.000 \AA$ \\
$R_{\mathrm{m}}$ & -0.40 \\
$a_{0}$ & $5.7347289 \AA$ \\
$a_{1}$ & $-255.0214692 \mathrm{~cm}^{-1}$ \\
$a_{2}$ & $-0.013405598929310479 \mathrm{~cm}^{-1}$ \\
$a_{3}$ & $0.153940442323125171 \times 10^{4} \mathrm{~cm}^{-1}$ \\
$a_{4}$ & $-0.626944977828736569 \times 10^{3} \mathrm{~cm}^{-1}$ \\
$a_{5}$ & $-0.147039918194012284 \times 10^{4} \mathrm{~cm}^{-1}$ \\
$a_{6}$ & $0.238628331428504282 \times 10^{3} \mathrm{~cm}^{-1}$ \\
$a_{7}$ & $-0.121465057044283844 \times 10^{4} \mathrm{~cm}^{-1}$ \\
$a_{8}$ & $-0.131024472517054273 \times 10^{5} \mathrm{~cm}^{-1}$ \\
$a_{9}$ & $0.410390478256789502 \times 10^{5} \mathrm{~cm}^{-1}$ \\
$a_{10}$ & $0.585609645570106004 \times 10^{5} \mathrm{~cm}^{-1}$ \\
$a_{11}$ & $-0.316660644987405278 \times 10^{6} \mathrm{~cm}^{-1}$ \\
$a_{12}$ & $0.178579875710784399 \times 10^{6} \mathrm{~cm}^{-1}$ \\
$a_{13}$ & $0.690085326716458891 \times 10^{6} \mathrm{~cm}^{-1}$ \\
$a_{14}$ & $-0.116538893384502688 \times 10^{7} \mathrm{~cm}^{-1}$ \\
$\mathrm{BO}_{\text {gen }}$ & $0.541518493723396794 \times 10^{6} \mathrm{~cm}^{-1}$ \\
$\mathrm{BO}_{\text {asym }}$ & -0.000465 \\
\hline \hline & $(-0.000001)$ \\
\hline
\end{tabular}


TABLE VIII. Feshbach resonances taken from Ref. [33] and their theoretical modeling. Columns o-c give the difference of observed field value (obs) and experimental uncertainty (unc) to the calculated one (not listed). The number indicates the model of evaluation as in Table II: (3) both $\mathrm{BO}$ corrections $\mathrm{BO}_{\text {gen }}$ and $\mathrm{BO}_{\text {asym }}$ for the homo- and heteronuclear cases.

\begin{tabular}{|c|c|c|c|c|c|c|c|c|c|}
\hline Isotope & $M$ & Atom pair & $l_{\min }$ & $l_{\max }$ & Type & Obs $[\mathrm{G}]$ & Unc $[\mathrm{G}]$ & $\mathrm{o}-\mathrm{c}(3)[\mathrm{G}]$ & \\
\hline \multirow[t]{12}{*}{$40 / 40$} & -6 & $|9 / 2,-7 / 2\rangle|9 / 2,-5 / 2\rangle$ & 0 & 0 & el & 228.8 & 0.4 & 0.131 & \\
\hline & -5 & $|9 / 2,-7 / 2\rangle|9 / 2,-3 / 2\rangle$ & 0 & 0 & el & 168.5 & 0.4 & -0.467 & \\
\hline & -5 & $|9 / 2,-7 / 2\rangle|9 / 2,-3 / 2\rangle$ & 0 & 0 & el & 260.3 & 0.6 & -0.128 & \\
\hline & -4 & $|9 / 2,-5 / 2\rangle|9 / 2,-3 / 2\rangle$ & 0 & 0 & el & 22.1 & 0.3 & 0.140 & \\
\hline & -4 & $|9 / 2,-5 / 2\rangle|9 / 2,-3 / 2\rangle$ & 0 & 0 & el & 178.0 & 1.0 & -0.017 & \\
\hline & -4 & $|9 / 2,-5 / 2\rangle|9 / 2,-3 / 2\rangle$ & 0 & 0 & el & 254.8 & 0.9 & -0.188 & \\
\hline & -2 & $|9 / 2,-3 / 2\rangle|9 / 2,-1 / 2\rangle$ & 0 & 0 & el & 37.2 & 0.3 & -0.199 & \\
\hline & -2 & $|9 / 2,-3 / 2\rangle|9 / 2,-1 / 2\rangle$ & 0 & 0 & el & 102.1 & 0.1 & -0.065 & \\
\hline & -2 & $|9 / 2,-3 / 2\rangle|9 / 2,-1 / 2\rangle$ & 0 & 0 & el & 138.2 & 0.1 & 0.034 & \\
\hline & -2 & $|9 / 2,-3 / 2\rangle|9 / 2,-1 / 2\rangle$ & 0 & 0 & el & 219.1 & 0.1 & -0.367 & \\
\hline & -2 & $|9 / 2,-3 / 2\rangle|9 / 2,-1 / 2\rangle$ & 0 & 0 & el & 292.3 & 0.4 & -0.180 & \\
\hline & 6 & $|9 / 2,7 / 2\rangle|9 / 2,5 / 2\rangle$ & 0 & 0 & el & 312.0 & 1.8 & -0.164 & \\
\hline \multirow[t]{21}{*}{$40 / 40$} & -7 & $|9 / 2,-9 / 2\rangle|9 / 2,-5 / 2\rangle$ & 1 & 1 & in & 215.0 & 5.0 & -1.060 & \\
\hline & -8 & $|9 / 2,-9 / 2\rangle|9 / 2,-5 / 2\rangle$ & 1 & 1 & in & 215.0 & 5.0 & -0.020 & \\
\hline & -6 & $|9 / 2,-5 / 2\rangle|9 / 2,-5 / 2\rangle$ & 1 & 1 & in & 232.8 & 0.2 & -0.141 & \\
\hline & -5 & $|9 / 2,-5 / 2\rangle|9 / 2,-5 / 2\rangle$ & 1 & 1 & in & 233.5 & 0.2 & -0.100 & \\
\hline & -5 & $|9 / 2,-5 / 2\rangle|9 / 2,-5 / 2\rangle$ & 1 & 1 & in & 245.3 & 0.4 & 0.019 & \\
\hline & -5 & $|9 / 2,-5 / 2\rangle|9 / 2,-3 / 2\rangle$ & 1 & 1 & in & 262.2 & 0.2 & 0.059 & \\
\hline & -4 & $|9 / 2,-5 / 2\rangle|9 / 2,-3 / 2\rangle$ & 1 & 1 & in & 262.6 & 0.2 & 0.140 & \\
\hline & -3 & $|9 / 2,-3 / 2\rangle|9 / 2,-3 / 2\rangle$ & 1 & 1 & in & 287.0 & 1.8 & -0.580 & \\
\hline & -3 & $|9 / 2,-3 / 2\rangle|9 / 2,-3 / 2\rangle$ & 1 & 1 & in & 311.8 & 0.4 & 0.090 & \\
\hline & -2 & $|9 / 2,-3 / 2\rangle|9 / 2,-1 / 2\rangle$ & 1 & 1 & in & 338.0 & 1.8 & -0.320 & \\
\hline & -1 & $|9 / 2,-1 / 2\rangle|9 / 2,-1 / 2\rangle$ & 1 & 1 & in & 373.0 & 1.8 & -0.654 & \\
\hline & 5 & $|9 / 2,5 / 2\rangle|9 / 2,5 / 2\rangle$ & 1 & 1 & in & 68.0 & 1.8 & 0.195 & \\
\hline & 5 & $|9 / 2,5 / 2\rangle|9 / 2,5 / 2\rangle$ & 1 & 1 & in & 102.0 & 1.8 & -0.581 & \\
\hline & 5 & $|9 / 2,5 / 2\rangle|9 / 2,5 / 2\rangle$ & 1 & 1 & in & 139.0 & 1.8 & 2.517 & \\
\hline & 5 & $|9 / 2,5 / 2\rangle|9 / 2,5 / 2\rangle$ & 1 & 1 & in & 324.0 & 1.8 & 0.238 & \\
\hline & 7 & $|9 / 2,5 / 2\rangle|9 / 2,9 / 2\rangle$ & 1 & 1 & in & 44.0 & 1.8 & -0.900 & \\
\hline & 6 & $|9 / 2,7 / 2\rangle|9 / 2,7 / 2\rangle$ & 1 & 1 & in & 43.8 & 0.2 & -0.011 & reassigned \\
\hline & 7 & $|9 / 2,7 / 2\rangle|9 / 2,7 / 2\rangle$ & 1 & 1 & in & 43.8 & 0.2 & 0.075 & reassigned \\
\hline & 8 & $|9 / 2,7 / 2\rangle|9 / 2,7 / 2\rangle$ & 1 & 1 & el & 44.7 & 0.2 & 0.100 & reassigned \\
\hline & 7 & $|9 / 2,7 / 2\rangle|9 / 2,7 / 2\rangle$ & 1 & 1 & in & 45.2 & 0.2 & 0.079 & reassigned \\
\hline & 7 & $|9 / 2,7 / 2\rangle|9 / 2,7 / 2\rangle$ & 1 & 1 & in & 46.4 & 0.2 & -0.254 & \\
\hline
\end{tabular}

\section{APPENDIX}

Tables V-VII show the potential parameters [defined in Eqs. (1), (3), and (4)] for the two states $X^{1} \Sigma_{g}^{+}$and $a^{3} \Sigma_{u}^{+}$, as derived during the evaluation. These results are improved potentials compared to the published ones [7], not only because the Feshbach data have been largely extended but also the number of potential parameters is significantly reduced, leading to a more stringent potential form with less danger of showing tiny oscillatory unphysical effects.

We analyzed the Feshbach resonances for ${ }^{40} \mathrm{~K}$ reported by Ludewig [33] using the derived potential system and the full beyond Born-Oppenheimer correction, model labeled by (3). The $s$-wave resonances are described consistently but some of the $p$-wave resonances needed a reassignment related to the assumed total magnetic quantum number $M$. The results are given in Table VIII. The $s$-wave resonances are dominated by the elastic channel but the $p$-wave resonances show mainly inelastic contributions as indicated in the column type by labels "el" or "in," respectively. The overall agreement is very good and confirms our analysis of the previously known resonances.

Furthermore, we analyzed the resonances given by Liu et al. [34]. With our model we confirm the positions of the three groups of $d$-wave Feshbach resonances; see Table IX. Our theoretical approach includes the effective spin-spin interaction, and thus we checked the splitting within the multiplet according the projection of rotational angular momentum $\mathrm{m}_{l}$ and found that the expected splitting in the case of $|1,1\rangle+$ $|1,0\rangle$ at $544 \mathrm{G}$ is too small to resolve the resonances $\mathrm{m}_{l}=0$ and \pm 1 in the study by Liu et al. [34]. This case is marked as "reassigned" in the table.

For the four reported groups of shape resonances, we see a systematic deviation from our predictions; compare the upper part of Table IX. The profile of these observed resonances is significantly asymmetric and highly dominated by the thermal distribution of the ensemble. Hence, a precise analysis of the resonance positions would require detailed knowledge of the experimental condition and the inclusion of their significant 
TABLE IX. Feshbach resonances taken from Ref. [34] and their theoretical modeling. Columns o-c give the difference of observed field value (obs) and experimental uncertainty (unc) to calculated one (not listed). The number indicates the model of evaluation as in Table II: (3) both $\mathrm{BO}$ corrections $\mathrm{BO}_{\text {gen }}$ and $\mathrm{BO}_{\text {asym }}$ for the homo- and heteronuclear cases. The upper panel summarizes the shape resonances (assuming $50 \mathrm{nK}$ ) and the lower one shows the Feshbach resonances (with $310 \mathrm{nK}$ according to Ref. [34]).

\begin{tabular}{lcccccccc}
\hline \hline Isotope & $M$ & Atom pair & $l_{\min }$ & $l_{\max }$ & Type & Obs [G] & Unc [G] & o-c(3) [G] \\
\hline $41 / 41$ & 2 & $|1,1\rangle|1,1\rangle$ & 0 & 2 & el & 16.83 & 0.04 & 0.539 \\
& 3 & $|1,1\rangle|1,1\rangle$ & 2 & 2 & el & 17.19 & 0.04 & 0.381 \\
& 4 & $|1,1\rangle|1,1\rangle$ & 2 & 2 & el & 18.75 & 0.04 & 0.333 \\
& 1 & $|1,1\rangle|1,0\rangle$ & 0 & 2 & in & 25.31 & 0.04 & 0.685 \\
& 2 & $|1,1\rangle|1,0\rangle$ & 2 & 2 & in & 25.41 & 0.23 & 0.642 \\
& 3 & $|1,1\rangle|1,0\rangle$ & 2 & 2 & in & 25.74 & 0.04 & 0.542 \\
& 0 & $|1,0\rangle|1,0\rangle$ & 0 & 2 & in & 63.30 & 0.04 & 0.806 \\
& 1 & $|1,0\rangle|1,0\rangle$ & 2 & 2 & in & 63.69 & 0.08 & 0.731 \\
& 2 & $|1,0\rangle|1,0\rangle$ & 2 & 2 & in & 65.16 & 0.06 & 0.694 \\
& -2 & $|1,-1\rangle|1,-1\rangle$ & 0 & 2 & in & 104.47 & 0.04 & 0.445 \\
& -1 & $|1,-1\rangle|1,-1\rangle$ & 2 & 2 & in & 104.95 & 0.04 & 0.475 \\
& 0 & $|1,-1\rangle|1,-1\rangle$ & 2 & 2 & in & 106.27 & 0.04 & 0.401 \\
& 2 & $|1,1\rangle|1,1\rangle$ & 0 & 2 & el & 530.48 & 0.04 & 0.122 \\
& 3 & $|1,1\rangle|1,1\rangle$ & 2 & 2 & el & 530.40 & 0.04 & 0.114 \\
& 4 & $|1,1\rangle|1,1\rangle$ & 2 & 2 & el & 530.18 & 0.04 & 0.131 \\
& 1 & $|1,1\rangle|1,0\rangle$ & 0 & 2 & el & 544.93 & 0.04 & 0.130 \\
& 3 & $|1,1\rangle|1,0\rangle$ & 2 & 2 & in & 544.79 & 0.04 & 0.140 \\
& 3 & $|1,1\rangle|1,0\rangle$ & 2 & 2 & in & 544.34 & 0.11 & -0.310 \\
& 1 & $|1,0\rangle|1,0\rangle$ & 0 & 2 & in & 565.23 & 0.04 & reassigned \\
& 2 & $|1,0\rangle|1,0\rangle$ & 2 & 2 & in & 565.05 & 0.04 & 0.053 \\
& $|1,0\rangle|1,0\rangle$ & 2 & 2 & in & 564.53 & 0.04 & 0.058 \\
\end{tabular}

kinetic energy dependence, which is not discussed in Liu et al. The authors applied an asymmetric line profile for the fit, which leads us to the assumption that the reported resonance positions are extrapolated to lower energies than the average value of $310 \mathrm{nK}$. We find better agreement with our predictions when the kinetic energy for our calculations is lowered to $50 \mathrm{nK}$; see Table IX. A conclusive analysis would require full access to the originally recorded spectra.
[1] M. Born and R. Oppenheimer, Ann. Phys. 389, 457 (1927).

[2] R. Herman and A. Asgharian, J. Mol. Spectrosc. 19, 305 (1966).

[3] J. K. G. Watson, J. Mol. Spectrosc. 80, 411 (1980).

[4] P. R. Bunker, J. Mol. Spectrosc. 68, 367 (1977).

[5] L. Che, Z. Ren, X. Wang, W. Dong, D. Dai, X. Wang, D. H. Zhang, X. Yang, L. Sheng, G. Li et al., Science 317, 1061 (2007).

[6] H. Knöckel, B. Bodermann, and E. Tiemann, Eur. Phys. J. D 28, 199 (2004).

[7] S. Falke, H. Knöckel, J. Friebe, M. Riedmann, E. Tiemann, and C. Lisdat, Phys. Rev. A 78, 012503 (2008).

[8] M. Fàrnìk, S. Davis, M. A. Kostin, O. Polyansky, J. Tennyson, and D. Nesbitt, J. Chem. Phys. 116, 6146 (2002).

[9] C. Chin, R. Grimm, P. Julienne, and E. Tiesinga, Rev. Mod. Phys. 82, 1225 (2010).

[10] C. A. Regal, C. Ticknor, J. L. Bohn, and D. S. Jin, Phys. Rev. Lett. 90, 053201 (2003).

[11] C. D'Errico, M. Zaccanti, M. Fattori, G. Roati, M. Inguscio, G. Modugno, and A. Simoni, New J. Phys. 9, 223 (2007).

[12] C.-H. Wu, I. Santiago, J. W. Park, P. Ahmadi, and M. W. Zwierlein, Phys. Rev. A 84, 011601(R) (2011).

[13] H.-Z. Chen, X.-C. Yao, Y.-P. Wu, X.-P. Liu, X.-Q. Wang, Y.-X. Wang, Y.-A. Chen, and J.-W. Pan, Phys. Rev. A 94, 033408 (2016).
[14] L. Tanzi, C. R. Cabrera, J. Sanz, P. Cheiney, M. Tomza, and L. Tarruell, Phys. Rev. A 98, 062712 (2018).

[15] J. H. van Vleck, J. Chem. Phys. 4, 327 (1936).

[16] T. A. Schulze, T. Hartmann, K. K. Voges, M. W. Gempel, E. Tiemann, A. Zenesini, and S. Ospelkaus, Phys. Rev. A 97, 023623 (2018).

[17] T. Hartmann, T. A. Schulze, K. K. Voges, P. Gersema, M. W. Gempel, E. Tiemann, A. Zenesini, and S. Ospelkaus, Phys. Rev. A 99, 032711 (2019).

[18] T. Weber, J. Herbig, M. Mark, H.-C. Nägerl, and R. Grimm, Phys. Rev. Lett. 91, 123201 (2003).

[19] P. D. Gregory, M. D. Frye, J. A. Blackmore, E. M. Bridge, R. Sawant, J. M. Hutson, and S. L. Cornish, Nat. Commun. 10, 3104 (2019).

[20] L. Fouché, A. Boissé, G. Berthet, S. Lepoutre, A. Simoni, and T. Bourdel, Phys. Rev. A 99, 022701 (2019).

[21] C. A. Regal and D. S. Jin, Phys. Rev. Lett. 90, 230404 (2003).

[22] C. Ticknor, C. A. Regal, D. S. Jin, and J. L. Bohn, Phys. Rev. A 69, 042712 (2004).

[23] C. A. Regal, M. Greiner, and D. S. Jin, Phys. Rev. Lett. 92, 040403 (2004).

[24] E. Arimondo, M. Inguscio, and P. Violino, Rev. Mod. Phys. 49, 31 (1977) 
[25] By sharing our manuscript with $\mathrm{M}$. Zwierlein [35], we learned that for the two resonances at lower fields, the authors extrapolated the field values from low-field calibrations and therefore an error of $2 \mathrm{G}$ is not unlikely. For the broad resonance at $542.7 \mathrm{G}$, an uncertainty of $0.5 \mathrm{G}$ would be a good estimate because an improved calibration was applied.

[26] At the bottom of Table I we give the deviations for the removed isotope combination derived from the three fit models. The magnitude of the deviations does not show a significant difference between the three models described, thus it is independent of type of modeling and not related to BO corrections.

[27] Note that within our many trials of nonlinear fitting we found one solution excluding $\mathrm{BO}$ corrections in which the $\sigma\left({ }^{39} \mathrm{~K}-{ }^{39} \mathrm{~K}\right)=0.924$ and $\sigma\left({ }^{39} \mathrm{~K}-{ }^{41} \mathrm{~K}\right)=0.988$ from the main body of data are almost equally distributed, but both are significantly larger than the ones derived from separate fits of the isotope combinations, namely 0.593 and 0.753 . Thus, also this fit shows the need for BO corrections.

[28] P. R. Bunker, J. Mol. Spectrosc. 46, 119 (1973).

[29] C. Amiot, J. Vergès, and C. Fellows, J. Chem. Phys. 103, 3350 (1995).

[30] A. Pashov, P. Popov, H. Knöckel, and E. Tiemann, Eur. Phys. J. D 46, 241 (2008).

[31] R. J. LeRoy, N. S. Dattani, J. A. Coxon, A. J. Ross, P. Crozet, and C. Linton, J. Chem. Phys. 131, 204309 (2009).

[32] M. Gerken, B. Tran, S. Häfner, E. Tiemann, B. Zhu, and M. Weidemüller, Phys. Rev. A 100, 050701(R) (2019).

[33] A. Ludewig, Ph.D. thesis, 2012, https://hdl.handle.net/11245/1. 369033.

[34] X.-P. Liu, X.-C. Yao, R. Qi, X.-Q. Wang, Y.-X. Wang, Y.-A. Chen, and J.-W. Pan, Phys. Rev. A 98, 022704 (2018).

[35] M. Zwierlein (private communication). 